\title{
Associações entre fatores socioeconômicos e desenvolvimento moral de adolescentes e a predisposição ao uso de substâncias psicoativas
}

\author{
Associations between socioeconomic factors and moral development of adolescents and \\ predisposition to the use of psychoactive substances \\ Associaciones entre factores socioeconómicos y desarrollo moral de los adolescentes y \\ predisposición al uso de substancias psicoativas
}

Recebido: 10/08/2021 | Revisado: 15/08/2021 | Aceito: 30/08/2021 | Publicado: 02/09/2021

\author{
Athos Ricardo Moraes Bastos Damasceno \\ ORCID: https://orcid.org/0000-0001-7012-4728 \\ Universidade do Estado do Pará, Brasil \\ E-mail: athos.damasceno@aluno.uepa.br \\ Adharsia Melissa Dias Marinho \\ ORCID: https://orcid.org/0000-0002-9773-5323 \\ Universidade do Estado Pará, Brasil \\ E-mail: adhmelissa@ hotmail.com \\ Dayane Diniz Martins \\ ORCID: https://orcid.org/0000-0002-6903-364X \\ Universidade do Estado do Pará, Brasil \\ E-mail: dayane.martins@ aluno.uepa.br \\ Laís Balla Lucena \\ ORCID: https://orcid.org/0000-0002-7068-9988 \\ Universidade do Estado do Pará, Brasil \\ E-mail: lais.lucena@aluno.uepa.br \\ Raul Aragão Martins \\ ORCID: https://orcid.org/0000-0001-6495-731X \\ Universidade Estadual Paulista, Brasil \\ E-mail: raul.martins@unesp.br \\ Ivete Furtado Ribeiro Caldas \\ ORCID: https://orcid.org/0000-0002-2095-101X \\ Universidade do Estado do Pará, Brasil \\ E-mail: ivetecaldas@uepa.br
}

\begin{abstract}
Resumo
A adolescência é o principal período em que o indivíduo constrói seus valores, julgamentos e ações. A vulnerabilidade inerente a fase de adolescência pode ser um risco para o uso de substâncias psicoativas (SPA's). Objetiva-se verificar associações entre fatores socioeconômicos e desenvolvimento moral de adolescentes e a predisposição ao uso de substâncias psicoativas. Participaram do estudo 100 adolescentes, entre 12 a 18 anos, de ambos os sexos e regularmente matriculados no ensino médio. Foram utilizados o questionário socioeconômico, CRAFFT/CESARE (acrônimo de Car; Relax; Alone; Forget; Family/Friends; Trouble) e o teste de competência moral. Os dados coletados foram analisados com o Teste Qui-quadrado $\left(\chi^{2}\right)$ de Pearson e o nível de significância escolhido foi de 0,05 . Quanto aos resultados encontrados, foi verificado que a maior parte da amostra tem entre 16-18 anos, são do sexo feminino, exercem função remunerada, praticam atividade física e alguma religião. No que tange a preferência dos estágios de desenvolvimento moral, no dilema do médico, houve predomínio dos estágios 1 e 2 , no do juiz, 5 e 6 e no dilema do operário, os estágios 4 e 5 . O nível mais elevado de moralidade sustenta a hipótese de a função remunerada é protetiva contra o uso de SPA's. Os adolescentes que praticam alguma religião demonstraram maior nível de competência moral em relação aos que não possuem religião, no dilema do operário, reafirmando o papel protetivo da espiritualidade contra a utilização de álcool e outras drogas.
\end{abstract}

Palavras-chave: Adolescente; Desenvolvimento moral; Questionário; Abuso de substâncias psicoativas.

\begin{abstract}
Adolescence is the main period in which individuals build their values, judgments and actions. The vulnerability inherent in the adolescence phase can be a risk for the use of psychoactive substances (SPA's). The objective is to verify associations between socioeconomic factors and the moral development of adolescents and the predisposition to the use of psychoactive substances. One hundred adolescents, aged between 12 and 18 years, of both sexes and regularly enrolled in high school participated in the study. The socioeconomic questionnaire, CRAFFT/CESARE (acronym for Car; Relax; Alone; Forget; Family/Friends; Trouble) and the moral competence test were used. The collected data were analyzed using Pearson's chi-square test $(\chi 2)$ and the chosen significance level was 0.05 . As for
\end{abstract}


the results found, it was found that most of the sample is aged between 16-18 years, are female, have a paid job, practice physical activity and some religion. Regarding the preference of the stages of moral development, in the physician's dilemma, there was a predominance of stages 1 and 2, in the judge, 5 and 6, and in the worker's dilemma, stages 4 and 5. The highest level of morality supports the hypothesis that the paid function is protective against the use of SPA's. Adolescents who practice a religion showed a higher level of moral competence compared to those who do not have a religion, in the worker's dilemma, reaffirming the protective role of spirituality against the use of alcohol and other drugs.

Keywords: Adolescent; Moral development; Quiz; Abuse of psychoactive substances.

\section{Resumen}

La adolescencia es el período principal en el que los individuos construyen sus valores, juicios y acciones. La vulnerabilidad inherente a la etapa de la adolescencia puede ser un riesgo por el uso de sustancias psicoactivas (SPA's). El objetivo es verificar asociaciones entre factores socioeconómicos y el desarrollo moral de los adolescentes y la predisposición al uso de sustancias psicoactivas. En el estudio participaron cien adolescentes, con edades comprendidas entre los 12 y los 18 años, de ambos sexos y matriculados habitualmente en el bachillerato. Se utilizó el cuestionario socioeconómico CRAFFT / CESARE (siglas de Car; Relax; Alone; Forget; Family / Friends; Trouble) y la prueba de competencia moral. Los datos recopilados se analizaron mediante la prueba de chi-cuadrado de Pearson $(\chi 2)$ y el nivel de significancia elegido fue 0,05 . En cuanto a los resultados encontrados, se encontró que la mayor parte de la muestra tiene entre 16 y 18 años, son mujeres, tienen un trabajo remunerado, practican actividad física y alguna religión. En cuanto a la preferencia de las etapas del desarrollo moral, en el dilema del médico predominaron las etapas 1 y 2 , en el juez, 5 y 6 , y en el dilema del trabajador, las etapas 4 y 5 . El nivel más alto de moralidad apoya la hipótesis de que la función paga protege contra el uso de SPA. Los adolescentes que practican una religión mostraron un mayor nivel de competencia moral en comparación con los que no tienen religión, en el dilema del trabajador, reafirmando el papel protector de la espiritualidad frente al consumo de alcohol y otras drogas.

Palabras clave: Adolescente; Desarrollo moral; Examen; Abuso de sustancias psicoactiva.

\section{Introdução}

O desenvolvimento humano se inicia na concepção e continua por toda a vida. O padrão é complexo por ser produto dos processos biológico, cognitivo e socioemocional, e pode ser influenciado por fatores biopsíquicos e socioculturais nos quais os adolescentes estão inseridos. Adolescência, do latim adolescere que significa crescer, desenvolver-se, tornar-se jovem, tem sido definida como um período biológico, psicológico e social compreendido entre os 10 e os 19 anos. Esse critério cronológico também tem sido adotado pelo Ministério da Saúde do Brasil (Who, 2018; Brasil, 1990; Marriel et al, 2013). No mundo, existe aproximadamente 1 bilhão de pessoas nessa faixa etária, representando cerca de $20 \%$ da população de todo o planeta (Freitas \& Sousa, 2020).

A adolescência é o principal período em que o indivíduo constrói seus valores, julgamentos e ações. Valores como justiça, dignidade, respeito, tolerância e igualdade podem ser aprendidos pelos jovens em todos os contextos e espaços onde convive e estabelece relações. A convivência com adultos e amigos e a observação do cotidiano contribuem para a formação moral do adolescente (Pratta \& Santos, 2007). Diversos fatores podem ser determinantes para o pleno desenvolvimento moral. Entre eles pode-se citar a idade, a educação, relacionamento com os pais e outros adultos com quem o adolescente convive e as condições socioeconômicas (Barros, 2020).

Dessa forma, a idade pode ser forte influência para a competência moral. Um estudo realizado com estudantes do curso de medicina demonstrou decréscimo de competência de juízo moral de alunos mais novos em relação a alunos mais velhos, podendo estar associado a exposição mais prolongada a fatores sociais e ambientes desfavoráveis ao desenvolvimento moral (Feitosa et al, 2013). Isso reforça, que a educação assume importante papel como formadora de princípios necessários para o bem-estar social geral e para solução de conflitos (Nunes \& Bataglia, 2019). As condições socioeconômicas desfavoráveis também representam um fator que pode influenciar (Martins, 2020), além dos relacionamentos sociais que são cruciais para a formação de senso moral no indivíduo. Durante a adolescência, os amigos são as figuras centrais e a satisfação das necessidades torna-se vital para o funcionamento da relação (Pagliace et al, 2019).

Nesse contexto, a vulnerabilidade inerente a fase de adolescência pode ser um risco para o uso de substâncias psicoativas (SPA's). O vício em SPA's é um distúrbio neuropsiquiátrico caracterizado por um desejo recorrente de continuar 
usando a droga, apesar das consequências prejudiciais. O uso/abuso de cocaína, crack, maconha, álcool e psicofármacos é considerado um problema de saúde pública com repercussões negativas de ordem psicológica (cognitiva e emocional), social, econômica, ocupacional e de saúde física (Zou et al., 2017). O uso dessas substâncias pode levar a repercussões em áreas límbicas e no centro de recompensa do cérebro, apresentando relação com agressividade, distúrbios comportamentais e de humor, alterações em memória, em funções executivas e na aprendizagem, sendo, portanto, importante fator de interferência na formação do senso crítico e julgamento moral nesse período da vida (Andrade et al., 2017).

Embora a maioria das pesquisas de desenvolvimento sobre o funcionamento cognitivo e psicossocial durante a adolescência envolva estudos psicológicos, estudos em neurociência esclarecem as bases neurais sobre essa fase da vida, incluindo os comportamentos típicos do adolescente, como comportamento de risco e impulsividade. Tais comportamentos são produtos da interação entre as mudanças de dois sistemas neurobiológicos distintos que estão em pleno amadurecimento nesse período da vida: o sistema socioemocional ou moral, localizado em áreas límbicas e paralímbicas do cérebro, e o sistema de controle cognitivo, que é composto principalmente pelos córtices parietal lateral e pré-frontal, responsável pela tomada de decisões e controle do impulso. Nesse período os circuitos neurais ficam mais eficientes e específicos, além de ocorrer um aumento significativo da dopamina, estimulando a busca de sensações em virtude do amadurecimento da via de recompensa (Martins et al, 2021). A via de recompensa está associada a comportamentos que geram sensação de prazer (p.ex. comer, beber, fazer sexo) e funciona como reforço positivo para que o comportamento seja repetido. Entretanto, outros estímulos também ativam a via de recompensa, p.ex. o uso de SPA's, mesmo quando associados com o risco de consequências negativas (Schlindwein-Zanini \& Sotili, 2019).

Dessa forma, a compreensão entre o desenvolvimento moral, a tomada de decisão e o uso de SPA's na adolescência é fundamental, pois em conjunto com as alterações corporais, o desenvolvimento cognitivo e emocional desses jovens sofre drásticas modificações nessa fase, colocando-o, muitas vezes, em risco (Carbonario, 2018). Dentro dessa perspectiva, este estudo tem como objetivo verificar associações entre fatores socioeconômicos e desenvolvimento moral de adolescentes e a predisposição ao uso de substâncias psicoativas.

\section{Metodologia}

Trata-se de um estudo quantitativo e transversal com amostra por conveniência. Os dados foram coletados na Escola Estadual de Ensino Médio Prof. Anízio Teixeira, no município de Marabá, Pará, localizado no sudeste paraense, distando cerca de 485 quilômetros da capital Belém e ocupando uma área de 15.128,058 quilômetros. O período de coleta de dados ocorreu nos meses de agosto a outubro de 2019.

Participaram do estudo 100 adolescentes regularmente matriculados no ensino médio. Foram incluídos alunos entre 12 a 18 anos, de ambos os sexos, que aceitaram participar da pesquisa assinando o Termo de Assentimento Livre e Esclarecido (TALE) e cujos responsáveis legais assinaram o Termo de Consentimento Livre e Esclarecido (TCLE). Foram excluídos alunos matriculados em outras escolas do município e que não estiveram presentes no dia da coleta de dados.

A pesquisa baseou sua metodologia na obra Metodologia Científica: Ciência, Ensino, Pesquisa. Editora Artes Médicas de Estrela, C (2018)

\subsection{Instrumentos}

Questionário socioeconômico: composto por 11 perguntas objetivas que acessavam informações sobre variáveis socioeconômicas dos estudantes (idade, sexo, se pratica exercícios físicos e religião, exerce de função remunerada e se pais e/ou responsáveis consomem bebidas alcoólicas), qualidade do relacionamento do estudante com seu responsável (pai e/ou mãe), e nível econômico familiar, baseado na Pesquisa de Orçamento Familiar (POF) do Instituto Brasileiro de Geografia e 
Estatística (IBGE) Esse instrumento objetiva avaliar a situação social e econômica dos estudantes e de sua família (CA et al, 2011).

Questionário CRAFFT/ CESARE (acrônimo de Car; Relax; Alone; Forget; Family/Friends; Trouble): utilizado na forma adaptada para o Brasil e internacionalmente recomendado para rastreamento de uso de substâncias psicoativas (SPA's) em adolescentes. Consiste num instrumento de triagem e não deve ser usado como critério diagnóstico. É composto por três perguntas de filtro sobre uso de bebidas alcoólicas, maconha ou outra droga (Parte A) e seis perguntas adicionais acerca de contexto e consequências relacionadas ao uso de SPAs (Parte B), cada resposta equivale a um ponto. Quanto a classificação de risco para o uso de SPAs é considerado CRAFFT/CESARE negativo (pontuação zero ou igual a um); positivo (maior ou igual a dois pontos); risco ao uso, abuso e dependência de SPA's (igual a 18 três pontos); suspeita de dependência de SPA's (maior ou igual a quatro) e risco de dependência de SPA's (igual a seis pontos) (Pereira, Schram, Azevedo, 2016).

Teste de Competência Moral (Moral Competence Teste - MCT): instrumento adaptado para versão brasileira que avalia a capacidade do sujeito em aplicar a estrutura de juízo moral em situações adversas, ou seja, o nível de competência moral dos participantes da pesquisa através do índice C. Três dilemas foram utilizados: Dilema do médico, juiz e operário, estes já validados nacional e internacionalmente. A estrutura dos três dilemas apresenta inicialmente uma pequena história sobre o assunto em questão, e o protagonista assume uma decisão para solucionar tal conflito em um respectivo contexto (Bataglia, Morais, Lepre, 2010; Bataglia, 2010; Lind, 2015). Logo após a leitura de cada dilema, o estudante era instruído a responder sua opinião sobre a decisão do protagonista. Suas respostas seguem um formato Likert de -3 a +3 , variando de forte discordância até forte concordância. Em seguida, no mesmo sentido, 12 argumentos, sendo seis favoráveis e seis contrários a ação do protagonista, eram também respondidos, no formato Likert de -4 a +4 , variando de rejeito completamente até aceito completamente o argumento.

\subsection{Procedimento}

Coleta de dados: Inicialmente foi solicitado à direção da escola que os pesquisadores participassem da reunião escolar de professores e responsáveis legais dos alunos, que ocorre bimestralmente de acordo com o calendário escolar, a fim de esclarecer os objetivos da pesquisa e a importância da participação dos alunos. Após assinatura no TCLE, os estudantes eram contatados e convidados a participarem da pesquisa respeitando as atividades escolares e o turno que estivessem matriculados.

Mediante a assinatura do TALE, iniciava-se a coleta de dados que foi conduzida em forma de entrevista individualizada. O pesquisador lia em voz compassada e em bom tom cada instrumento, e as perguntas relacionadas, o participante respondia oralmente. O pesquisador registrava assinalando com um " $\mathrm{X}$ " sobre a resposta indicada.

Uma sala foi disponibilizada nas dependências da escola para melhor comodidade e privacidade durante as entrevistas. A sala era arejada, com boa luminosidade, medindo $3 \times 5 \mathrm{~m}$, aproximadamente, e continha 5 cadeiras e 1 mesa.

Primeiramente, foi coletado informações do CRAFFT/CESARE, seguido pelo teste de competência moral, e por fim, o questionário socioeconômico. Após a entrevista cada estudante foi identificado com um número (em substituição ao nome do aluno) que foram armazenados em um envelope. O tempo de cada entrevista foi de 30 minutos.

Análise de dados. Para análise de dados, inicialmente foi realizado o cálculo do Índice C que representa o nível de competência moral para cada sujeito, seguido do cálculo da média para cada grupo do estudo. Os resultados das características socioeconômicas e sociodemográficas dos participantes do estudo foram descritos, pela frequência e porcentagem (Tabela 1). Os dados coletados foram analisados com o Teste Qui-quadrado $(\chi 2)$ de Pearson e o nível de significância escolhido foi de 0,05 . 
Inicialmente foram computadas a frequência absoluta e relativa do perfil socioeconômico e a avaliação do CRAFFT/CESARE dos estudantes. Em seguida, foi calculado a preferência por estágio do desenvolvimento moral e o Índice C (nível de competência moral) para cada sujeito, e em seguida, o índice C médio para cada dilema. Os valores das variações de significância entre as médias dos Índice C total são 5 (alta significância) e 10 (muito alta significância). E a escala de referência corresponde de 0-9 pontos como competência moral muito baixa; 10-29 pontos para competência média (intervalo no qual se inserem a maioria dos indivíduos), e acima de 30 pontos para alta competência moral (LIND, 2000).

Os resultados das características socioeconômicas e sociodemográficas dos participantes do estudo foram descritos pela frequência e porcentagem (Tabela 1). Os dados coletados foram analisados com o Teste Qui-quadrado ( $\chi 2)$ de Pearson e o nível de significância escolhido foi de 0,001. A utilização desse teste estatístico nos permitiu analisar possíveis associações entre as variáveis qualitativas desse estudo.

\section{Resultados}

A tabela 1 detalha as características socioeconômicas dos estudantes do estudo. Quanto a idade 72 (72.0\%) tem entre 16 e 18 anos de idade, $51(51.0 \%)$ são do sexo feminino, 23 (23.0\%) exercem função remunerada, 73 (73.0\%) praticam atividade física e $81(81.0 \%)$ alguma religião. A maioria dos pais ou responsáveis dos adolescentes 51 (51.0\%) consume bebidas alcoólicas e 90 (90.0\%) relatam ter bom relacionamento bom com a mãe, entretanto, com o pai, apenas 66 (66.0\%). Quanto a classificação socioeconômica, as classes B2 e C1 prevalecem com 30 (30.0\%) e 20 (20.0\%), respectivamente.

Tabela 1 - Características socioeconômicas dos adolescentes do estudo.

\begin{tabular}{|c|c|c|}
\hline \multirow[b]{2}{*}{ Variáveis } & \multicolumn{2}{|c|}{$\mathbf{N}=\mathbf{1 0 0}$} \\
\hline & $\mathbf{n}$ & $\%$ \\
\hline \multicolumn{3}{|l|}{ Idade } \\
\hline $12-15$ & 28 & 28.0 \\
\hline $16-18$ & 72 & 72.0 \\
\hline \multicolumn{3}{|l|}{ Sexo } \\
\hline Feminino & 51 & 51.0 \\
\hline Masculino & 49 & 49.0 \\
\hline \multicolumn{3}{|l|}{ Prática de exercícios físicos } \\
\hline Sim & 73 & 73.0 \\
\hline Não & 27 & 27.0 \\
\hline \multicolumn{3}{|l|}{ Função remunerada } \\
\hline Sim & 23 & 23.0 \\
\hline Não & 77 & 77.0 \\
\hline \multicolumn{3}{|l|}{ Religião } \\
\hline Sim & 81 & 81.0 \\
\hline Não & 19 & 19.0 \\
\hline \multicolumn{3}{|c|}{ Bebidas alcoólicas por pais ou responsáveis } \\
\hline Sim & 51 & 51.0 \\
\hline Não & 49 & 49.0 \\
\hline \multicolumn{3}{|l|}{ Relacionamento com a mãe } \\
\hline Não tenho mãe & 1 & 1.0 \\
\hline Bom & 90 & 90.0 \\
\hline
\end{tabular}




$\begin{array}{lll}\text { Regular } & 8 & 8.0 \\ \text { Ruim } & 0 & 0.0 \\ \text { tenho contato } & 1 & 1.0\end{array}$

Relacionamento com o pai

$\begin{array}{ccc}\text { Não tenho pai } & 6 & 6.0 \\ \text { Bom } & 66 & 66.0 \\ \text { Regular } & 17 & 17.0 \\ \text { Ruim } & 5 & 5.0 \\ \text { Não tenho contato } & 6 & 6.0\end{array}$

Classificação da classe socioeconômica

\begin{tabular}{ccc} 
A & 14 & 14.0 \\
B1 & 19 & 19.0 \\
B2 & 30 & 30.0 \\
C1 & 20 & 20.0 \\
C2 & 12 & 12.0 \\
D - E & 5 & 5.0 \\
\hline
\end{tabular}

Fonte: Dados da pesquisa.

Em relação ao CRAFFT/CESARE para rastreamento de uso de SPA's, a maioria dos adolescentes $72(72.0 \%)$ apresentou risco negativo, todavia 11 (11.0\%) apresentam risco para o uso, abuso ou dependência de SPA's, 6 (6.0\%) suspeita de dependência de SPA's e $2(2.0 \%)$ risco de dependência de SPA's. No que se refere à preferência por estágios do desenvolvimento moral no dilema do médico, houve predomínio do estágio 2 (36\%), seguido pelo estágio 1 (23\%) e menor preferência pelo estágio 5 (4\%). No dilema do juiz, os estágios 5 e 6 destacaram-se com $23 \%$ e $25 \%$, respectivamente. E no dilema do operário, o estágio 4 foi o mais predominante com 42\%, seguido pelo estágio 3, com 20\% (Figura 1). Nossos resultados também mostram que o Índice C médio dos dilemas do médico, juiz e operário correspondeu a 31.94, 35.87, 41.83 pontos, respectivamente. A média geral foi 38.55 pontos. 
Figura 1 - Porcentagem da preferência por estágios do desenvolvimento moral referente ao dilema do Médico (A), Juiz (B) e Operário (C).

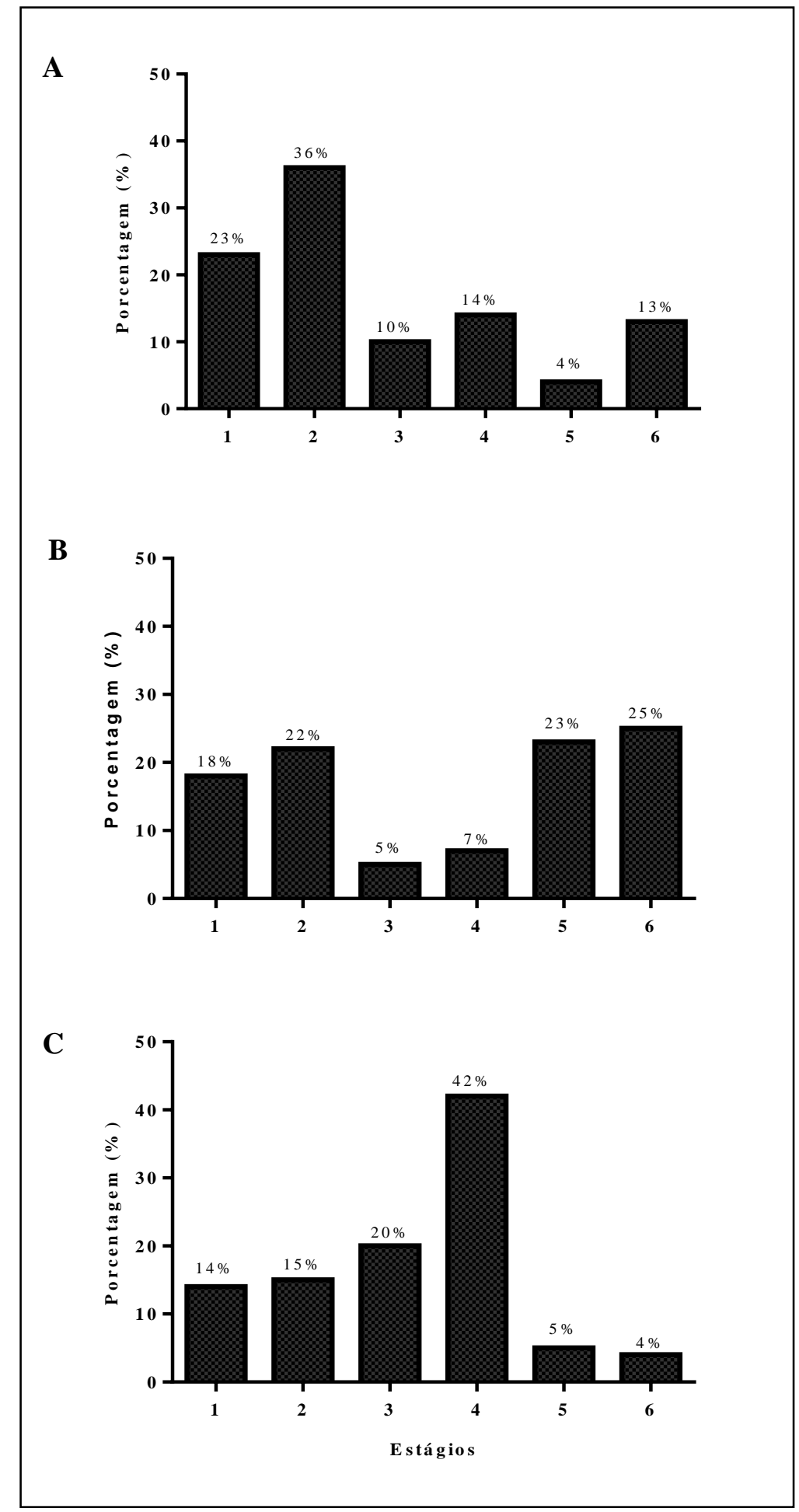

Fonte: Dados da pesquisa

A análise mostrou efeito de relação significativa apenas entre as variáveis socioeconômicas (idade, função remunerada e religião) e o nível de competência moral do sujeito. Entre as demais variáveis não houve significância. A variável idade mostrou relação com o nível de competência moral do dilema do Médico e do Juiz. Treze (100\%) dos estudantes entre 16 a 18 anos apresentaram nível baixo e 17 (32.1\%) nível alto ( $\chi 2=9.28$, df $=2, p<0.01)$. Já no dilema do Juiz, 31 
Research, Society and Development, v. 10, n. 11, e307101119286, 2021

(CC BY 4.0) | ISSN 2525-3409 | DOI: http://dx.doi.org/10.33448/rsd-v10i11.19286

(86.1\%) dos estudantes entre 16 a 18 anos apresentaram nível médio, enquanto que 21 (35.6\%) daqueles entre 12 a 15 anos, nível alto $(\chi 2=5,67, \mathrm{df}=1, p<0.017)$ (Tabela 2$)$.

Tabela 2 - Variável idade e o nível de competência moral do dilema do médico e do juiz.

\begin{tabular}{|c|c|c|c|c|c|}
\hline \multirow[b]{2}{*}{ Idade (anos) } & \multicolumn{4}{|c|}{ Nível de Competência Moral } & \multirow[b]{2}{*}{$p$ valor $^{(1)}$} \\
\hline & $\begin{array}{l}\text { Baixo } \\
n(\%)\end{array}$ & $\begin{array}{c}\text { Médio } \\
n(\%)\end{array}$ & $\begin{array}{l}\text { Alto } \\
n(\%)\end{array}$ & $\begin{array}{l}\text { Total } \\
N(\%)\end{array}$ & \\
\hline \multicolumn{6}{|c|}{ Dilema do Médico } \\
\hline $12-15$ & $0(0.0)$ & $11(32.3)$ & $17(32.1)$ & $28(28.0)$ & $<0.01 *$ \\
\hline $16-18$ & $13(100.0)$ & $23(67.7)$ & $36(67.9)$ & $72(72.0)$ & \\
\hline \multicolumn{6}{|c|}{ Dilema do Juiz } \\
\hline $12-15$ & $0(0.0)$ & $5(13.9)$ & $21(35.6)$ & $26(27.4)$ & $<0.017 *$ \\
\hline $16-18$ & $0(0.0)$ & $31(86.1)$ & $38(64.4)$ & $69(72.6)$ & \\
\hline
\end{tabular}

(1) Teste Qui-quadrado de Pearson para tendência ( $p$ valor <0.01).

** Valores Altamente significativos; *Valores Significativos Fonte: Dados da pesquisa.

A variável função remunerada também houve relação significativa com o nível de competência moral do dilema do juiz. Treze (36.1\%) dos estudantes que possuíam função remunerada apresentaram nível competência moral médio, enquanto aqueles que não possuíam, apresentaram nível alto $(\chi 2=6.44$, df $=1, p<0.011)$ (Tabela 3$)$.

Tabela 3 - Variável função remunerada e o nível de competência moral do dilema do juiz.

\begin{tabular}{|c|c|c|c|c|c|}
\hline \multirow[b]{2}{*}{ Função remunerada } & \multicolumn{4}{|c|}{ Nível de Competência Moral } & \multirow[b]{2}{*}{$p$ valor $^{(1)}$} \\
\hline & $\begin{array}{l}\text { Baixo } \\
n(\%)\end{array}$ & $\begin{array}{c}\text { Médio } \\
n(\%)\end{array}$ & $\begin{array}{c}\text { Alto } \\
n(\%)\end{array}$ & $\begin{array}{l}\text { Total } \\
N(\%)\end{array}$ & \\
\hline Sim & $0(0.0)$ & $13(36.1)$ & $8(13.5)$ & $21(22.1)$ & $<0.011^{*}$ \\
\hline Não & $0(0.0)$ & $23(63.9)$ & $51(86.5)$ & $74(77.9)$ & \\
\hline
\end{tabular}

(1) Teste Qui-quadrado de Pearson para tendência ( $p$ valor $<0.01)$.

** Valores Altamente significativos; *Valores Significativos. Fonte: Dados da pesquisa.

Já a variável religião mostrou relação significativa com o nível de competência moral do dilema do juiz. Sessenta e um $(87.2 \%)$ dos estudantes que se autodeclararam ter religião apresentaram nível de competência moral alto, enquanto 11 $(37.9 \%)$ daqueles que não possuíam, apresentaram nível médio $(\chi 2=7.42$, df $=1, p<0.007)$ (Tabela 4). 
Tabela 4 - Variável religião e o nível de competência moral do dilema do operário.

\begin{tabular}{|c|c|c|c|c|c|}
\hline \multicolumn{6}{|c|}{ Nível de Competência Moral } \\
\hline \multirow{2}{*}{ Religião } & Baixo & Médio & Alto & Total & \multirow{2}{*}{$p$ valor $^{(1)}$} \\
\hline & $n(\%)$ & $n(\%)$ & $n(\%)$ & $N(\%)$ & \\
\hline Sim & $0(0.0)$ & $18(62.1)$ & $61(87.2)$ & $79(79.8)$ & $<0.007 *$ \\
\hline Não & $0(0.0)$ & $11(37.9)$ & $9(12.8)$ & $20(20.2)$ & \\
\hline
\end{tabular}

(1) Teste Qui-quadrado de Pearson para tendência ( $p$ valor <0.01).

** Valores Altamente significativos; *Valores Significativo. Fonte: Dados da pesquisa.

\section{Discussão}

A literatura aponta que há um aumento proporcional entre idade e consumo de SPA's, principalmente após os 15 anos. Isso pode ser explicado pela sede de novas experiências, típica dessa fase (Andrade et al., 2017; HORTA et al., 2018). A ingesta de álcool é uma prática, de certa forma, bem aceita e estimulada por grande parte da sociedade, e é compreensível a curiosidade do adolescente de aventurar-se em algo "tão adulto", mas, o consumo excessivo de álcool pode ser preditor ao uso de SPA's nesse grupo etário (Raposo Et Al., 2017).

Nossos resultados mostram que a maioria dos adolescentes entrevistados praticam atividade física e alguma religião, sendo estas práticas considerada como protetivas contra o uso de SPA's, podendo justificar o número baixo de estudantes que acusaram já ter usado SPA's (Santos Et Al, 2015; Nasser Et Al, 2016; Raposo, 2017; Benincasa Et Al, 2017; Jorge Et Al, 2018). Em relação a prática religiosa, por oferecer um sistema de condutas morais que visam, teoricamente, respeitar a vida, o corpo, o mundo e a sociedade, atribui-se baixo índice do uso de SPA's em pessoas ligadas a algum tipo de fé (Motyka Et Al, 2021; Diniz Et Al, 2020; Raposo Et Al., 2017; Benincasa Et Al, 2017; Santos Et Al., 2015; Gomes Et Al, 2015).

Em nosso estudo, mesmo com mais da metade dos pais e/ou responsáveis utilizando bebidas alcoólicas, a maioria dos adolescentes não apresentou fator de risco para o uso ou abuso de SPA's, mostrando que o bom relacionamento com a família, em especial os pais e/ou responsáveis é importante para a proteção do adolescente contra o uso de SPA's, corroborando com nossos resultados em que a maioria relatou ter bom relacionamento com pais e/ou mãe (Motyka Et Al, 2021; Chein Et Al, 2011; Paiva Et Al, 2015; Andrade Et Al, 2017; Horta Et Al, 2018; Malta Et Al, 2018).

Quanto a classificação socioeconômica as classes mais inferiores prevaleceram, em virtude do estudo ter sido realizado em uma escola pública. A classe econômica do indivíduo pode ser um fator precursor, mas não determinante, do uso de SPA's. O uso de álcool, outras drogas na adolescência e a classe socioeconômica não são consoantes quanto a associação entre menos privilégios e mais drogas. Na Europa, a variação dessa relação entre os países é imensa, e essa relação pode ser válida apenas quando o nível de pobreza é extremo e está ligado a problemas de comportamento, como o furto, por exemplo (Martins-Oliveira Et Al, 2016; Hormenu Et Al, 2018; Shackleton Et Al, 2019).

No que se refere ao Índice $\mathrm{C}$ médio, todos os dilemas (médico, juiz e operário) apresentaram nível alto de competência moral (acima de 30 pontos). Esse padrão na pontuação do Índice C médio corrobora com a de outros países envolvendo também dilemas da Eutanásia e do Juiz (quantidade de vidas vs. respeito a uma vida). Uma pesquisa realizada na China encontrou Índice C médio de 31,4 pontos e na Alemanha, em torno de 40. Diferente nos EUA, que apresentou 23,8 pontos e no Irã, 20 (Liquat, 2012; Akin, 2018).

Outros resultados apontam que a houve relação significativa entre a variável idade e os dilemas do médico e do juiz. A maioria dos adolescentes entre 12-15 anos inclui-se em um nível de competência moral alto, diferente daqueles mais velhos, entre 16-18 anos que apresentaram níveis médio e alto. Conforme a teoria do raciocínio moral de Kohlberg é adequado que a maior parte dos adolescentes esteja em um nível médio de moralidade, o nível convencional, em que o indivíduo age de acordo 
com as expectativas das pessoas que o cercam, principalmente das autoridades, obedecendo regras tão somente por obedecer, sem julgamento crítico. Entretanto, na amostra vê-se a preponderância de adolescentes no nível pós-convencional ou alto em que o ser humano se guia por valores morais e éticos universais, independente do ambiente em que se encontram (Bataglia Et Al, 2010). O predomínio de adolescentes inseridos no nível pós-convencional concorda com Twenge (2017), que afirma que a as gerações mais novas possuem um nível de empatia e tolerância maior para com o seu próximo, o que também explicaria o porquê da maior proporção de participantes mais novos no nível alto em relação ao grupo de 16-18 anos.

A idade consiste em um indicador-chave para julgamentos morais. Para Kohlberg os indivíduos podem desenvolver um amadurecimento moral no decurso da vida. Todavia, no estudo de Feitosa et al. (2013) os resultados mostram menor nível de competência de moral entre os sujeitos mais velhos. Os autores sugerem que esse processo de erosão da competência moral, provavelmente, seja decorrente da exposição mais prolongada a fatores sociais e a ambientes “desfavoráveis”. É provável que a menor competência de juízo moral entre estudantes mais velhos faça parte de um processo de erosão da competência moral, decorrente da exposição mais prolongada a fatores sociais e a ambientes "desfavoráveis", todavia, necessitando de mais esclarecimentos científicos.

Quanto à função remunerada, a maioria dos estudantes não exerce nenhuma atividade com retorno financeiro. Isso já era previsível na amostra, já que a maior parte dos trabalhadores formais no Brasil têm idade entre 25 e 59 anos (IBGE,2020). Dentre os adolescentes que têm função remunerada apenas 8 entrevistados apresentaram alto nível de competência moral no Dilema do Juiz, sustentando-se a hipótese de que o consumo de SPA's nessa amostra seja menos prevalente (IBGE,2020).

O Dilema do Juiz envolve aspectos cognitivos e afetivos com enfoque na dúvida entre quantidade de vida versus respeito a uma vida. Acredita-se que o jovem que tem a vivência da atividade profissional apresenta um maior ciclo de convívio que envolve situações de conflito, cobranças, administração de tempo, relacionamentos de hierarquias do que o jovem que não exerce atividade laboral, fortalecendo, assim, o resultado da pesquisa, já que essa amostra apresentou alto desempenho no teste de competência moral nesse dilema em específico (Rizzo Et Al, 2010).

Por outro lado, no Brasil, há raros estudos que associam o trabalho remunerado entre os adolescentes com o uso de SPA's. Dentre os mais relevantes (2.472 estudantes), tem-se uma avaliação realizada em 2007, com estudantes da cidade de Cuiabá - MT, no qual houve maiores prevalências entre os estudantes trabalhadores para o uso de álcool e tabaco. Entre as justificativas para esse consumo estão o contato com pessoas mais velhas no ambiente de trabalho, renda disponível aumentada e carga horária estressante, a qual pode ser uma motivação para apoiar este uso de drogas (Souza, Filho, 2007).

Em um outro estudo, o consumo de bebidas alcoólicas foi 1,33 maior entre adolescentes com trabalho remunerado, o mesmo foi observado em uma pesquisa realizada no Estado da Bahia, em que também houve associação positiva com o padrão de consumo de álcool mais frequente entre adolescentes que trabalhavam. Além disso, não só o consumo, mas também a intensidade do consumo é maior nesses jovens, caracterizando-os como binge drinking, ou seja, têm o hábito de beber em grande quantidade (Veiga Et Al, 2016; Raposo Et Al, 2017; Gomes Et Al, 2019).

Por fim, a variável religião mostrou relação com o nível de competência moral do dilema do operário. Dos que apresentaram nível de competência moral alta a maioria dos adolescentes declararam possuir religião e dentre os que declararam não possuir religião, a maioria apresentou nível de competência moral média. Os resultados encontrados para o dilema do operário apontaram que o estágio 3 foi o de maior escolha entre os estudantes, inserindo-os, de acordo com a Teoria de Kohlberg no nível convencional, no qual a maioria dos adolescentes deve estar incluída. Nesse estágio, o indivíduo tem como base para suas decisões as regras do grupo social e não o pensamento egocêntrico apoiado em interesses próprios. Assim, a religião pode ter uma influência no nível de competência moral.

Um estudo realizado por Santos et al (2015) demonstrou que os adolescentes que não possuem uma religião ou têm e não praticam uma religião possuem mais chances de consumir álcool, cigarros, drogas ilícitas e ter um número elevado de 
parceiros sexuais, quando comparados aos que possuem e praticam uma determinada religião. A prática de uma religião, independentemente da religião seguida, foi considerada como fator de proteção para o consumo de álcool, cigarros, drogas ilícitas e número elevado de parceiros sexuais. Ademais, segundo Gomes et al (2015), tanto a prática religiosa quanto o consumo de drogas interferem intensamente na saúde física e mental do adolescente e, além disso, têm grande impacto sobre seus comportamentos de risco e seu desenvolvimento psicossocial.

\section{Conclusão}

Nossos resultados mostraram que a maior parte da amostra tem entre 16-18 anos, são do sexo feminino, exercem função remunerada, praticam atividade física e alguma religião. A maioria dos pais e/ou responsáveis destes consomem bebidas alcoólicas e possuem boa relação com os filhos, principalmente a figura materna. As classes econômicas mais inferiores prevalecem entre os entrevistados.

O Índice C Médio de todos os dilemas foi alto, corroborando com outros estudos semelhantes ao redor do mundo. No que tange a preferência dos estágios de desenvolvimento moral, no dilema do médico, houve predomínio dos estágios 1 e 2 , no do juiz, 5 e 6 e no dilema do operário, os estágios 4 e 5 . Os adolescentes mais jovens apresentaram níveis morais mais altos que os mais velhos. O nível mais elevado de moralidade sustenta a hipótese de a função remunerada é protetiva contra o uso de SPA's, entretanto, a literatura precisa de mais trabalhos que analisem esta temática. Além disso, os adolescentes que praticam alguma religião demonstraram maior nível de competência moral em relação aos que não possuem religião, no dilema do operário, reafirmando o papel protetivo da espiritualidade contra a utilização de álcool e outras drogas.

Portanto, considera-se que esta pesquisa tem bastante valor para os estudos relacionados ao uso de SPA's, desenvolvimento moral e neurociência, pois ajuda a reconhecer e entender os fatores protetivos e de risco para a utilização destas substâncias, dessa forma, auxiliando a tomada de decisões familiares, políticas escolares e públicas em vista de um melhor e mais seguro desenvolvimento dos jovens.

Como sugestão para as próximas produções científicas que abordarão essa temática, propõe-se que os estudantes de escola privada sejam também incluídos na amostra, para mais correlações entre fatores socioeconômicos, desenvolvimento moral de adolescentes e o uso de substâncias psicoativas.

\section{Referências}

Andrade, M. E. D., Santos, I. H. F., Souza, A. A. M. D., Silva, A. C. S., Leite, T. D. S., Oliveira, C. C. D. C., \& Albuquerque Júnior, R. L. C. D. (2017). Experimentação de substâncias psicoativas por estudantes de escolas públicas. Revista de saúde pública, $51,82$.

Barros, L. S. (2020). Adolescência, desenvolvimento moral e educação: considerações a partir do desenho animado caverna do dragão. Aprender - Caderno De Filosofia E Psicologia Da Educação, (24), 245-261.

Bataglia, P. U. R., Morais, A. D., \& Lepre, R. M. (2010). A teoria de Kohlberg sobre o desenvolvimento do raciocínio moral e os instrumentos de avaliação de juízo e competência moral em uso no Brasil. Estudos de Psicologia (Natal), 15(1), 25-32.

Benincasa, M., Resende, M. M., Heleno, M. G. V., \& Custódio, E. M. (2017). Qualidade de vida e resiliência em estudantes do ensino médio: um estudo epidemiológico na cidade de São Paulo. Psicologia Revista, 26(2), 363-383.

Chein, J., Albert, D., O’Brien, L., Uckert, K., \& Steinberg, L. (2011). Peers increase adolescent risk taking by enhancing activity in the brain's reward circuitry. Developmental Science, 14 (2), 1-10.

Diniz, A.P, Minucci, G.S., Roama-Alves, R.J, Souza e Souza, L.P. Espiritualidade e Religiosidade como práticas de enfrentamento ao uso abusivo de Drogas. Revista Psicologia, Diversidade e Saúde, n. 9, v.1, p. 88-102, 2020.

Emerson, T. L., Conroy, S. J., Stanley, C. W. Ethical attitudes of accountants: Recent evidence from a practitioners' survey. Journal of Business Ethics, v. 71, n. 1, p. 73-87, 2007.

Estrela, C. Metodologia Científica: Ciência, Ensino, Pesquisa. Editora Artes Médicas, 2018

Feitosa, H.N., Rego, S., Bataglia, P., Rego, G., Nunes, R. Competência de juízo moral dos estudantes de medicina: um estudo piloto. Rev. bras. educ. med. [online]. 2013, vol.37, n.1 [cited 2021-04-07], pp.5-14. 
Felipe, A.O.B., Carvalho, A.M.P, Andrade, C.U.B. Espiritualidade e religião como protetores ao uso de drogas em adolescente. Rev. Eletrônica Saúde Mental Álcool Drog. (Ed. port.), vol.11, n.1, pp. 49-58, 2015.

Gomes, M. B. Rezende, M. M. Custódio, E. M. Heleno, M. G. V. Serafim, A. P. David, V. F. Adolescência, drogas e religiosidade no município de São Paulo - Brasil. Bol. psicol [online]. 2015, vol.65, n.142, pp. 1-13.

IBGE - Instituto Brasileiro De Geografia E Estatística. Pesquisa Nacional por Amostra de Domicílios Contínua, 2020.

Horta, R. L., Mola, C. L., Horta, B. L., Mattos, C. N. B., Andreazzi, M. A. R., Oliveira- Campos, M., \& Malta, D. C. (2018). Prevalence and factors associated with illicit drug use throughout life: National School Health Survey 2015. Revista Brasileira de Epidemiologia, 1 (21).

Hormenu, T., Júnior, J. E. H., \& Schack, T. (2018). Predictors of alcohol consumption among inschool adolescents in the Central Region of Ghana: A baseline information for developing cognitive-behavioural interventions. PLoS One,13 (11)..

Jorge, K. O., Ferreira, R. C., Ferreira, E. F., Kawachi, I., Zarzar, P. M., \& Pordeus, I. A. (2018). Peer group influence and illicit drug use among adolescente students in Brazil: a cross-sectional study. Caderno de Saúde Pública, 3 (34).

Lind, G. (2000). O significado e medida da competência moral revisitada: um modelo do duplo aspecto da competência moral. Revista Psicologia: Reflexão e Crítica, 13 (3), 399416

Malta, D. C., Machado, I. E., Felisbino-Mendes, M. S., Prado, R. R., Pinto, M. A. S., Oliveira-Campos, M., Souza, M. F. M., \& Assunção, A. A. (2018). Use of psychoactive substances among Brazilian adolescents and associated factors: National School-based Health Survey, 2015. Revista Brasileira de Epidemiologia, (1).

Martins, M. O. Desenvolvimento moral e marginalização social: julgamento moral de crianças de bairros marginalizados. Revista Eletrônica de Psicologia e Epistemologia Genéticas. Volume 12 Número 1, Jan-Jul/2020.

Martins, D. D. Damasceno, A. R. M. B. Lucena, L. B. Siqueira, H. Tadano, Y. S. Caldas, I. F. R. Clusterização do perfil de adolescentes escolares com predisposição ao uso de substância psicoativas. Research, Society and Development, v. 10, n. 2, e37510212528, 2021 (CC BY 4.0).

Martins-Oliveira, J.G., Ferreira, R.C., Vale, M.P., Ferreira, E. F., \& Zarzar, P. (2016). Risco De Dependência Do Álcool: Prevalência, Problemas Relacionados e Fatores Socioeconômicos. Revista Ciência e Saúde Coletiva, 21 (1), 17-26.

Motyka, M.A; Al-imam, A. Representations of Psychoactive Drugs' Use in Mass Culture and Their Impact on Audiences. Int. J. Environ. Res. Public Health, 2021. Doi: $10.3390 /$ ijerph 18116000

Nasser, R. L., Branco, J. C., Lara, D. R., Del Vecchio, F. B., Wienner, C., Mesquita, P., Souza, L.D.M., Silva, R. A., \& Jansen, K. (2016). Atividade física de lazer e uso de substâncias lícitas em uma amostra populacional de adultos jovens. Ciência \& Saúde Coletiva, 21 (1).

Nunes, V. B; Bataglia, P. U. R. Trabalho com dilemas morais na resolução de conflitos entre escolares: fundamentos habermasianos à teoria kohlberguiana. Revista de Educação PUC-Campinas, v.24, n.1, p.125-138, 2019.

Pagliace, A. G. S., Maftum, M. A., Lacerda, M. R., Kantorski, L. P., Nimtz M. A., \& Brusamarello T. (2019). Avaliação do cuidado às crianças e aos adolescentes usuários de substâncias psicoativas: potencialidades e fragilidades. Texto \& Contexto Enfermagem, 2019, 18 (3).

Paiva, P. C. P., Paiva, H. N., Lamounier, J. A., Ferreira, E. F., César, C. A. S., \& Zarzar, P. M. (2015). Consumo de álcool em binge por adolescentes escolares de 12 anos de idade e sua associação com sexo, condição socioeconômica e consumo de álcool por melhores amigos e familiares. Revista Ciência e Saúde Coletiva, 20 (11), 3427-3435.

Pratta, E. M. M. Santos, M. A. Família e adolescência: a influência do contexto familiar no desenvolvimento psicológico de seus membros. Psicol. estud. [online]. 2007, vol.12, n.2 pp.247-256. ISSN 1807-0329.

Pereira, D. R. Morais, A. Desenvolvimento moral: o que a educação infantil tem a ver com isso? Revista Eletrônica de Psicologia e Epistemologia Genéticas. 2016, vol.8, n.1.

Raposo, J. C. S., Costa, A.C.Q., Valença, P. A. M., Zarzar, P. M., Diniz, A. S., Colares, V., \& Franca, C. (2017). Uso de drogas ilícitas e binge drinking entre estudantes adolescentes. Revista de Saúde Pública, 51 (83).

Rizzo, C. B. S; Chamon, E. M. Q. O. O sentido do trabalho para o adolescente trabalhador. Trab. educ. saúde (Online), Rio de Janeiro, v. 8, n. 3, p. 407-417, Nov. 2010

Santos, A.R.M., Oliveira, L. M. F. T., Júnior, J. C. F., Silva, P. P. C., Silva, E. A. P. C., \& Freitas, C. M. S. M. (2015). Associação entre prática religiosa e comportamentos de risco à saúde em adolescentes de Pernambuco. Revista Brasileira de Atividade Física e Saúde, 20 (3), 284-296.

Shackleton, N., Milne, B.J., \& Jerrim, J. (2019). Socioeconomic Inequalities in Adolescent Substance Use: Evidence from Twenty-Four European Countries. Substance Use and Misuse, 54(6), 1044-1049.

Schlindwein-Zanini, R., Sotili, M. Uso de drogas, repercussões e intervenções neuropsicológicas. Cadernos Brasileiros de Saúde Mental, ISSN 1984-2147, Florianópolis, v.11, n.28, p.94-116, 2019.

Souza, D. P. Filho, D. X. Uso recente de álcool, tabaco e outras drogas entre estudantes adolescentes trabalhadores e não trabalhadores. Rev. bras. epidemiol., São Paulo, v. 10, n. 2, p. 276-287, June 2007.

Twenge, J. M. I.Gen: Why Today's Super-Connected Kids Are Growing Up Less Rebellious, More Tolerant, Less Happy--and Completely Unprepared for Adulthood--and What That Means for the Rest of Us. Atria Books, 2017. 
Research, Society and Development, v. 10, n. 11, e307101119286, 2021

(CC BY 4.0) | ISSN 2525-3409 | DOI: http://dx.doi.org/10.33448/rsd-v10i11.19286

Veiga, L. D. B., Santos, V. C., Santos, M. G., Ribeiro, J. F., Amaral, A. S. N., Nery, A. A., Casotti, C. A. Prevalência e fatores associados à experimentação e ao consumo de bebidas alcoólicas entre adolescentes escolares. Cad. saúde colet., Rio de Janeiro, v. 24, n. 3, p. 368-375, Sept. 2016.

Zou, Z., Wang, H., d'Oleire Uquillas, F., Wang, X., Ding, J., \& Chen, H. (2017). Definition of Substance and Non-substance Addiction. Advances in experimental medicine and biology, 1010, 21-41. 